\title{
Avaliação fitoterápica da Jatropha gossypiifolia L. na cicatrização de suturas na parede abdominal ventral de ratos $^{1}$
}

\author{
Phytotherapic evaluation of Jatropha gossypiifolia L. on rats ventral abdominal wall wound \\ healing
}

José Ulcijara Aquino ${ }^{2}$, Nicolau Gregori Czeczko ${ }^{3}$, Osvaldo Malafaia ${ }^{3}$, Ulrich Andreas Dietz ${ }^{3}$, Jurandir Marcondes Ribas Filho $^{3}$, Paulo Afonso Nunes Nassif ${ }^{3}$, Ubirajara Araújo ${ }^{4}$, João Boroncello ${ }^{4}$, Manoel Francisco da Silva Santos ${ }^{2}$, Eduardo Antonio de Andrade Santos ${ }^{6}$

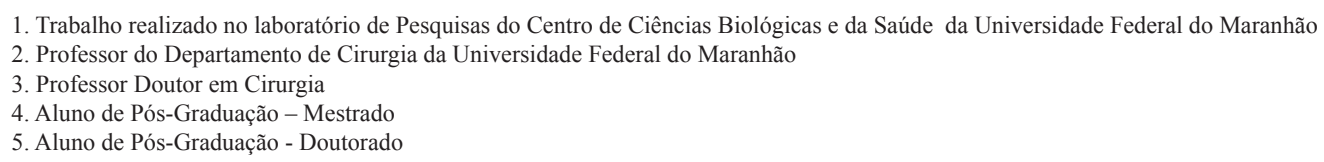

\section{RESUMO}

Introdução: A espécie vegetal Jatropha gossypiifolia L., conhecida também como pião roxo, é utilizada na medicina popular como cicatrizante, anti-hipertensivo, purgativo e diurético. Objetivo: Avaliar a influência da administração intraperitoneal da Jatropha gossypiifolia L., na cicatrização de suturas da parede abdominal ventral de ratos, observando-se os seus aspectos macroscópicos, tensiométricos e microscópicos. Métodos: Foram utilizados no procedimento 40 ratos da linhagem Wistar, machos, distribuídos em dois grupos de 20. Após incisão da parede e abertura da cavidade abdominal, foi instilado $1 \mathrm{ml} / \mathrm{kg} /$ peso de cloreto de sódio a $0,9 \%$ no grupo controle e no grupo Jatropha o extrato bruto etanólico da Jatropha gossypiifolia L., na concentração de $1 \mathrm{ml} / \mathrm{kg} /$ peso. Realizou-se a sutura da parede abdominal com fio de polipropileno, com pontos separados. Os animais foram avaliados na sua evolução pós-operatória e mortos em dois subgrupos, no $3^{\circ}$ e $7^{\circ}$ dia. Analisou-se a parede abdominal ventral macroscopicamente, mediu-se a força de resistência a tensão e foram estudados os aspectos histológicos do reparo cicatricial. Resultados: No exame macroscópico encontraram-se aderências mais intensas nos subgrupos Jatropha no $3^{\circ}$ e $7^{\circ}$ dia. A avaliação tensiométrica foi significantemente maior nos subgrupos Jatropha no $3^{\circ}$ e $7^{\circ}$ dia. A avaliação histológica comparativa entre os subgrupos demonstrou que o processo inflamatório agudo foi significantemente maior no subgrupo Jatropha no $3^{\circ}$ e $7^{\circ}$ dia; a neoformação capilar foi significantemente maior no $3^{\circ}$ dia pós-operatório do subgrupo Jatropha sendo os outros parâmetros histológicos semelhantes. Conclusão: O uso do extrato bruto de Jatropha gossypiifolia L. intraperitoneal não demonstrou melhora significativa no processo de cicatrização da sutura da parede abdominal ventral de ratos com a dose e concentração utilizadas.

Descritores: Jatropha gossypiifolia L. Pião Roxo. Tua-tua. Parede Abdominal. Cicatrização de Feridas. Ratos Wistar.

\begin{abstract}
Introduction: The Jatropha gossypiifolia L., which is used in popular medicine is considered to have good diuretic effect in hypertension and is also used as a laxative drug. It seems to have a healing effect, although not proved till now. Purpose: To evaluate the influence of intraperitoneum administration of Jatropha Gossypiifolia L., in suture healing of ventral abdominal wall of rats, through tensiometric measurement, macro and microscopic aspect of post-operative period. Methods: Forty wistar male rates were allocated in two groups of 20 animals. After the incision and exposure of abdominal cavity $1 \mathrm{ml} / \mathrm{kg} /$ weight of $0,9 \%$ sodium chloride solution was injected in control group, and in the other one the injection was of $1 \mathrm{ml} / \mathrm{kg} /$ weight of a gross ethanol extract of Jatropha gossypiifolia L. The suture of the abdominal wall was than performed with polypropylene separated stitches. The animals were followed-up and killed in the third and seventh days. The ventral abdominal wall was macroscopically analyzed, the resistance strength to strain was measured and it was also studied the histological aspects. Results: On macroscopic examination more intense adhesion was found on the group of Jatropha in both third and seventh post-operative days. The strain evaluation was meanly greater on Jatropha group also in third and seventh days. Conclusion: The histological comparative analysis between the different groups showed that the acute inflammatory process was meanly greater for the Jatropha group in third and seventh post-operative days. The vascular neoformation was significantly greater in third pos-operative day of Jathopha group; the other histological parameters were just alike. The intraperitoneum injection of Jatropha extract did not have any significant improvement for the wound healing on ventral abdominal wall on the evaluated animals in this study, no matter if analyzed at the third or seventh pos-operative days.
\end{abstract}

Key words: Jathopha gossypiifolia L. Bellyache bush. Tua-tua. Abdominal. Cicatrization. Rats. Medicine Cathastique. 


\section{Introdução}

A cicatrização constitui um processo biológico complexo que envolve inflamação, quimiotaxia, proliferação celular, diferenciação e remodelação, sendo essencial para manter a integridade do organismo. A melhor técnica operatória para o fechamento da parede abdominal permanece como objetivo a ser alcançado pelo cirurgião $0^{1,2,3,4}$. Ainda há dúvidas sobre os aspectos do uso de substâncias químicas e/ou procedimentos que possam agilizar ou retardar o processo, sejam eles na ferida limpa ou infectada. Várias substâncias já foram usadas e testadas no processo de cicatrização, como o açúcar, óleo de rosa-mosqueta, uso tópico de papaína, insulina, Aloe vera (babosa) e extrato alcoólico de flores de ixora que que atua na cicatrização estimulando fatores que aumentam a granulação ${ }^{1}$.

Apesar do desenvolvimento nas áreas de síntese orgânica, biologia molecular e biologia industrial, parte dos fármacos permanece sendo obtida a partir de matérias-primas vegetais. É notório que no Brasil e outros países em desenvolvimento, as plantas medicinais são muito utilizadas no tratamento das doenças mais prevalentes. A validação científica desses produtos é essencial ao uso como medicamentos comprovadamente efetivos e estudos farmacológicos, de toxicidade, controle de qualidade e de resultado terapêuticos devem ser estimulados 5 .

As espécies de plantas do gênero Jatropha (Euphorbiaceae) são conhecidas popularmente como pião-roxo, jalapão, raiz-do-téu, batata-do-téu, erva-purgante, mamoninha e tua-tua. No conhecimento folclórico, o gênero Jatropha é utilizado no tratamento de alguns processos patológicos como reumatismo e hidropsias, neoplasias e úlceras, além de ser empregado como diurético, antidiarréico e antihipertensivo ${ }^{6}$.

$\mathrm{Na}$ literatura consultada não se encontra relato sobre o uso do extrato de Jatropha gossypifolia L. como cicatrizante em animais de experimentação.

Assim, este trabalho objetiva estudar comparativamente a ação do uso intraperitoneal da Jatropha gossypiifolia L. na cicatrização de suturas realizadas na parede abdominal ventral de ratos.

\section{Métodos}

Este trabalho foi realizado no Laboratório de Pesquisa do Departamento de Fisiologia e Farmacologia da Universidade Federal do Maranhão. Foram respeitadas todas as normas de experimentação animal e princípios éticos em experimentação animal do Colégio Brasileiro de Experimentação Animal - COBEA. Este trabalho foi aprovado pelo Comitê de Ética em Pesquisa do Hospital de Clínicas da Universidade Federal do Maranhão.

Utilizaram-se 40 ratos (Rattus Norvergicus albinus) de linhagem Wistar, machos, com peso variando entre 95 e 140 gramas no início do experimento. Os animais foram distribuídos aleatoriamente em dois grupos contendo 20 ratos cada, denominados de grupo controle (GC) e grupo Jatropha (GJ). No grupo controle (GC) foi instilado $1 \mathrm{ml} /$ $\mathrm{kg} /$ peso de cloreto de sódio a $0,9 \%$ na cavidade peritoneal e no grupo Jatropha (GJ) instilou-se o extrato bruto etanólico de Jatropha gossypiifolia L., na concentração de 100mg/ml sendo administrada a dose de $100 \mathrm{mg} / \mathrm{kg}$ de peso .

\section{Procedimento operatório}

Todos os animais submeteram-se ao mesmo procedimento cirúrgico que consistiu de: a) anestesia geral com éter sulfúrico; b) fixação em prancha cirúrgica própria para esses animais; c) epilação do abdome e anti-sepsia com povinilpirrolidona-iodo; d) incisão de $3 \mathrm{~cm}$ na linha média da parede abdominal; e) abertura e inspeção da cavidade abdominal; f) instilação da solução fitoterápica ou solução salina conforme o grupo em estudo (Figura 1); g) síntese da parede abdominal com pontos separados de fio de polipropileno 5-0 (Prolene ${ }^{\circledR}$, Ethicon); h) síntese da pele com sutura contínua de polipropileno 5-0.

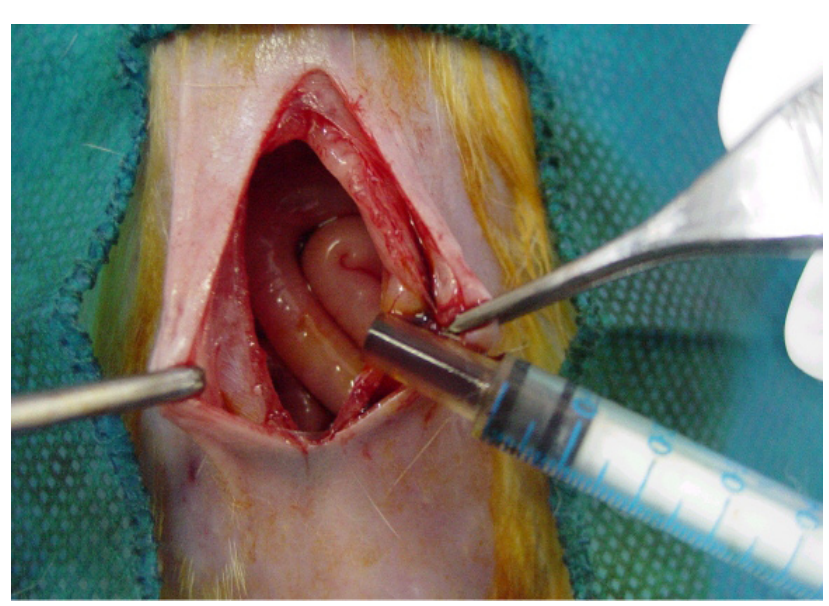

FIGURA 1 - Colocação do cloreto de sódio a $0,9 \%$ ou extrato bruto etanólico de Jatropha gossypiifolia L. na cavidade peritoneal

Ao final do procedimento, cada grupo (GC e GJ) foi dividido, aleatoriamente em dois subgrupos com 10 animais cada, conforme o dia da morte programada. Os ratos dos subgrupos controle SGC3 e Jatropha SGJ3 foram sacrificados no terceiro dia do período pós-operatório e aqueles dos subgrupos controle SGC7 e Jatropha SGJ7, no sétimo dia do pós-operatório.

Marcou-se os animais individualmente com ácido pícrico para identificação dos grupois e em seguida foram colocados nas gaiolas identificadas conforme o subgrupo nas mesmas condições do período pré-operatório.

Os animais foram alimentados com ração padrão para ratos no período pós-operatório imediato e examinados diariamente, verificando-se as condições comportamentais e aspecto da ferida operatória preenchendo-se os dados em protocolo padrão para todos.

Na sacrificação dos animais utilizou-se dose letal de éter sulfúrico por via inalatória.

Após a morte, tiveram a ferida operatória examinada para detectar se havia presença de infecção, deiscência, 
hematoma ou seroma. Após retirada da pele, a parede abdominal ventral foi exposta. Realizou-se a ressecção da parede abdominal expondo-se assim a cavidade peritoneal. Avaliou-se a presença ou não de aderências entre a sutura da parede abdominal e as vísceras abdominais. As aderências foram avaliadas segundo critérios de Nair ${ }^{7}$. Observou-se a presença ou não de infecção intra-peritoneal. Na retirada da parede abdominal ventral do animal, a peça foi dividida em duas partes de tamanhos diferentes. A parte cranial, recortada em formato retangular (Figura 2), tinha a dimensão maior $45 \mathrm{~mm}$ e no seu centro $15 \mathrm{~mm}$. A sutura da parede abdominal estava no centro. Este segmento foi acondicionado em recipiente com cloreto de sódio a $0,9 \%$ e transportado para o Laboratório de Mecânica do Centro Federal de Educação Tecnológica do Maranhão, para o estudo tensiométrico. (Figura 2)

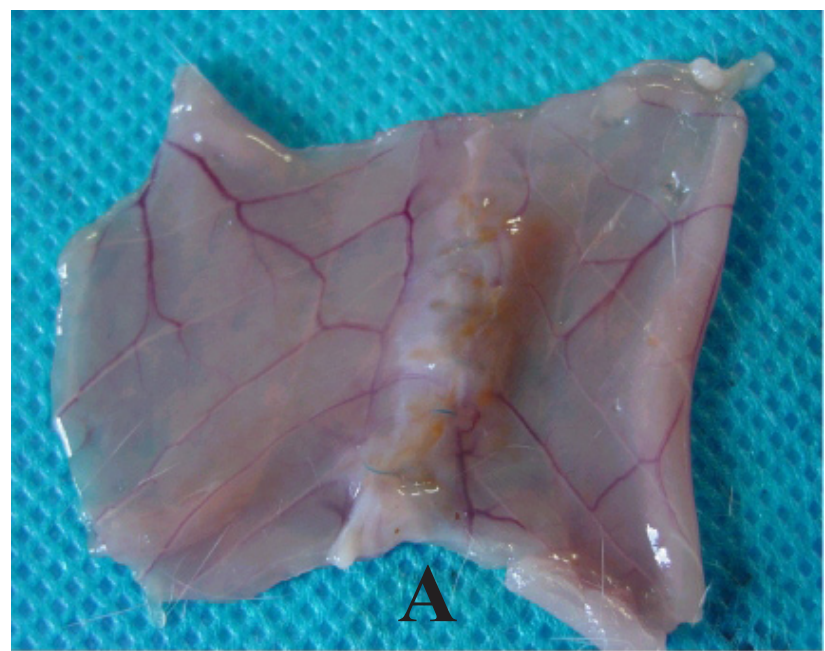

FIGURA 2 - Peça operatória em forma retangular para estudo tensiométrico. Observa-se em A o local da sutura realizada

A porção caudal tinha $20 \mathrm{~mm}$ de comprimento por 5 mm de largura, estando a sutura no seu ponto médio. Este fragmento era preso em placas de isopor e colocado em formol a $10 \%$. As peças eram encaminhadas ao processamento histológico.

\section{Estudo tensiométrico}

No estudo tensiométrico foi utilizada uma máquina universal de ensaio (modelo TT 2420, Tira Test Maschinenbau Gmbh - Germany) do Laboratório de Ensaios Mecânicos do Centro Federal de Educação Tecnológica do Maranhão. Essa máquina é equipada com uma célula de carga com capacidade máxima de $20 \mathrm{k}$ Newton e possui precisão de $0,5 \%$. As deformações foram medidas através do deslocamento do carro superior do equipamento não havendo escorregamento da amostra das garras de fixação (Figura 3).

Após a fixação das amostras nos acessórios da máquina universal de ensaios, elas foram submetidas à tração axial. Foram obtidos gráficos de carga e deformação em cada ensaio, sendo calculadas as propriedades mecânicas. Os gráficos resultantes ilustram como foi determinada essa propriedade, sendo que LM representa o limite máximo, Cmáx a carga máxima e Dmáx a deformação na carga máxima sofrida pela amostra. O limite máximo (LM) é o maior valor da carga e da deformação observada em cada ensaio, até o rompimento da amostra. Carga é a força aplicada durante a realização do ensaio, para promover a ruptura da amostra ensaiada.

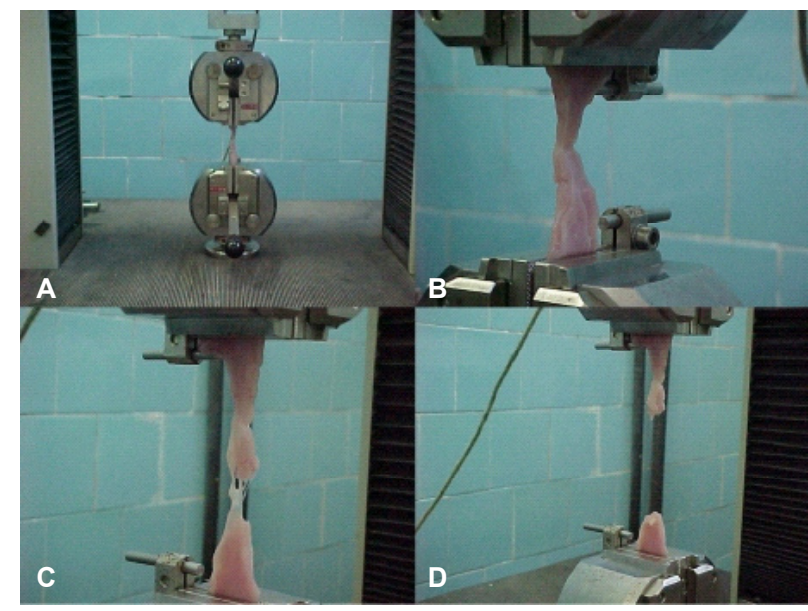

FIGURA 3 - Teste de ruptura; A - Peça cirúrgica tensionada; B - Início da medida da tensão; C - Ruptura da peça; D - Fragmentos rotos da peça cirúrgica

\section{Avaliação microscópica}

O processamento histológico foi o habitual e a análise das lâminas foram realizados no Laboratório de Patologia do Hospital Universitário da UFMA. A coloração foi a de hematoxilina-eosina.

A análise dos cortes histológicos foi realizada, sem o conhecimento prévio do grupo e subgrupo animal a que pertencia. Os critérios histológicos incluíram: inflamação aguda, inflamação crônica, necrose isquêmica, reação gigantocelular do tipo corpo estranho, proliferação fibroblástica, fibrose (colagenização), coaptação das bordas da sutura e neoformação capilar.

\section{Resultados}

$\mathrm{O}$ ato operatório de todos os animais transcorreu sem complicações. Todos os animais recuperaram-se bem da anestesia, durante o período pós-operatório não houve intercorrências. Os animais de ambos os grupos ganharam peso durante todo o estudo.

\section{Avaliação macroscópica}

A incidência de infecção da parede abdominal está demonstrada em detalhes na Tabela 1. Observa-se que houve 
$10 \%$ de contaminação da ferida operatória não demonstrando diferença com significância estatística entre os grupos.

Na Tabela 1, observa-se a incidência dos graus das aderências dos quatro grupos determinada pelo escore de Nair, onde se observa significância estatística para análise intergrupo favorável do GJ na avaliação de 3 dias.

TABELA 1 - Distribuição dos animais segundo o critério de aderência de Nair e a significância do teste de Mann-Withney para comparação inter-subgrupos (SGC3 × SGJ3)

\begin{tabular}{lccccc}
\hline & SGC3 & SGJ3 & SGC7 & SGJ7 & TOTAL \\
\hline $\mathbf{0}$ & 10 & 4 & 8 & 5 & 27 \\
$\mathbf{1}$ & 0 & 5 & 1 & 2 & 8 \\
$\mathbf{2}$ & 0 & 1 & 1 & 3 & 5 \\
\hline TOTAL & 10 & 10 & 10 & 10 & 40 \\
\hline \multicolumn{5}{c}{$\mathrm{p}=0,023$} & $\mathrm{p}=0,023$ \\
\hline
\end{tabular}

\section{Estudo tensiométrico}

No Figura 4 observa-se a análise intergrupo entre SGC3 / SGJ3 e SGC7 / SGJ7 da variável CMAX. Foi observada diferença estatística no $7^{\circ}$ dia pós-operatório favorável ao GJ.

\section{Avaliação microscópica}

No $3^{\circ}$ dia do período pós-operatório a reação inflamatória aguda foi classificada como discreta em cinco animais no SGC3, moderada em três e acentuada em seis no SGJ3. A diferença entre o grau de inflamação aguda do SGJ3 e SGC3 foi estatisticamente significativa (Figura 5). No $7^{\circ}$ dia, a reação inflamatória aguda foi classificada como discreta em dois animais do SGC7, discreta em três, moderada em dois e acentuada em dois animais no SGJ7. O SGJ7 apresentou maior proporção de animais com inflamação, discreta, moderada e acentuada. Houve diferença estatisticamente significante entre os subgrupos.

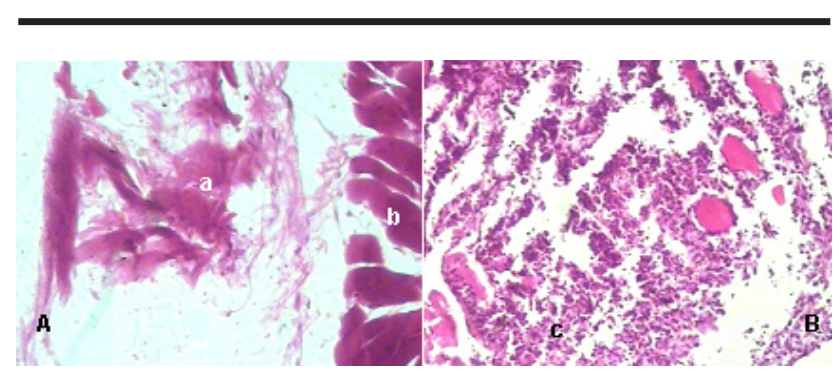

FIGURA 5 - Fotomicrografia de inflamação aguda na parede abdominal ventral de ratos mortos no $3^{\circ}$ dia do período pós-operatório. Nota: A - Rato do subgrupo SGC3, lâmina corada pela técnica hematoxilina-eosina, aumento de 40X. B - Rato do subgrupo SGJ3, lâmina corada pela técnica hematoxilina-eosina, aumento de 40X. a - Tecido conjuntivo normal. b - Tecido muscular da parede abdominal. c - Neutrófilos

As diferenças da reação inflamatória crônica, necrose isquêmica, reação gigantocelular, reação gigantocelular, fibrose (colagenização) e coaptação de bordas da sutura, não foram significativas.

No $3^{\circ}$ dia do período pós-operatório a neoformação capilar foi classificada como discreta em oito animais, moderada em um e ausente em um no SGC3 e discreta em dois animais e moderada em oito animais no SGJ3. No $7^{\circ}$ dia do período

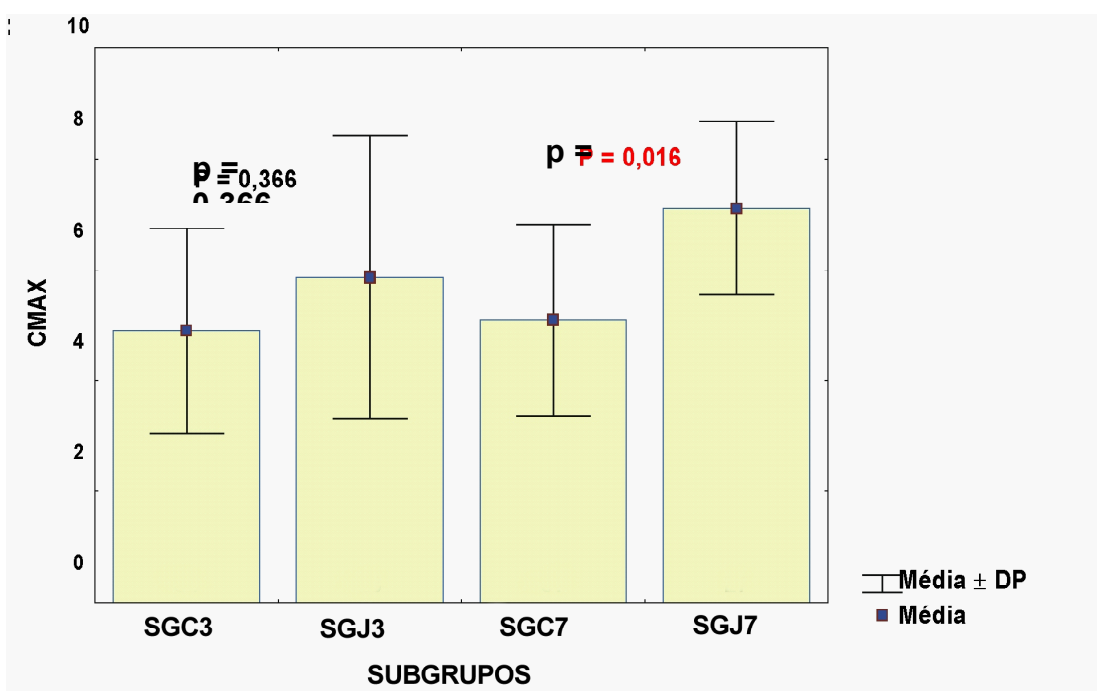

FIGURA 4 - Média e desvio-padrão da carga máxima (CMAX) dos grupos controle (GC) e Jatropha (GJ)e a significância do teste $\mathrm{t}$ da análise inter-grupo. Legenda: CMAX - Carga máxima. SGC3 - Subgrupo controle $3^{\circ}$ dia. SGJ3 - Subgrupo Jatropha $3^{\circ}$ dia. SGC7 - Subgrupo controle $7^{\circ}$ dia. SGJ7 - Subgrupo Jatropha $7^{\circ}$ dia. 
pós-operatório foi discreta em três animais, moderada em dois e ausente em cinco, no SGC7. Por sua vez ela, foi moderada em quatro animais, discreta em quatro e ausente em dois animais no SGJ7. Houve diferença estatisticamente significante no subgrupo Jatropha na comparação do $3^{\circ}$ dia do período pós-operatório. (Figura 6)

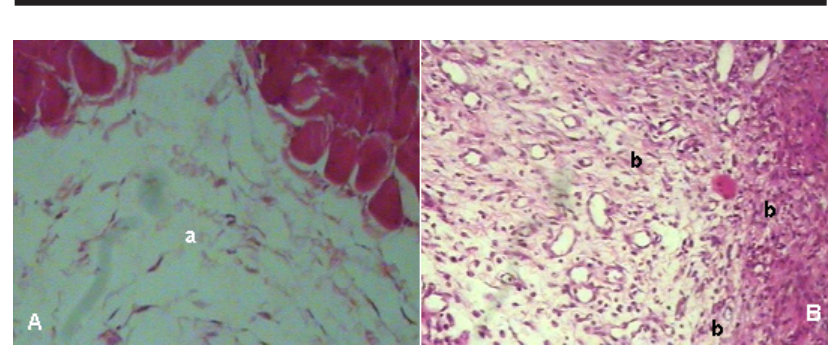

FIGURA 6 - Fotomicrografia de neoformação capilar na parede abdominal ventral de ratos mortos no $3^{\circ}$ dia do período pós-operatório. Nota: A - Rato do subgrupo SGC3, lâmina corada pela técnica hematoxilina-eosina, aumento de 40X. B - Rato do subgrupo SGJ3, lâmina corada pela técnica hematoxilinaeosina, aumento de 40X. a - Ausência de neoformação vascular. b - Neoformação vascular.

\section{Discussão}

A Organização Mundial de Saúde estima que 80\% da população dos países em desenvolvimento tratam-se com medicamentos de medicina popular e, desse total, $85 \%$ usam produtos de origem vegetal.

O extrato etanólico de Jatropha gossypiifolia L. foi escolhido por ser inédito em estudo de cicatrização em animais, não havendo na literatura consultada qualquer trabalho sobre esse tema.

O pião roxo, Jatropha gossypiifolia L., é planta vastamente usada pela medicina popular do Brasil e de outros países, principalmente os latino-americanos e africanos, para diversos fins terapêuticos, predominando o uso com fim anti-hipertensivo ${ }^{3}$.

O rato da linhagem Wistar foi o animal escolhido devido à facilidade de aquisição e manipulação, tamanho (pequeno porte), acomodação, resistente às agressões cirúrgicas e aos processos infecciosos e com baixa mortalidade ${ }^{7}$. Foram utilizados ratos machos, pois, segundo Teves et al. ${ }^{8}$ variações dos ciclos hormonais das fêmeas poderiam interferir no mecanismo do processo de reparação tecidual. Até o final do experimento, não ocorreram complicações pós-operatórias, tampouco relevantes variações de peso tendo todos os animais sobrevivido.

O rato tem sido utilizado como padronizado para estudos de cicatrização especialmente da parede abdominal $1^{5,9,10,11}$.

O fio de polipropileno monofilamentado apresenta- se como ideal em vários estudos ${ }^{6}$. Neste estudo, os ratos evoluíram sem discência, corroborando a segurança de seu emprego. Houve infecção em 10\% dos animais, limitada à superfície.

Utilizou-se a laparotomia mediana para o estudo da cicatrização com o fitoterápico $^{10,11,12}$. A via de administração intraperitoneal foi determinada pela facilidade e eficiência na absorção de substância ali empregadas.

Os animais evoluíram sem complicações importantes. $\mathrm{O}$ método adotado neste experimento utilizou o princípio de aplicação de carga através de esforços estáticos da tração, para a obtenção dos parâmetros que avaliam o material em estudo, dando um indicativo da sua resistência aos esforços nele aplicados.

Os parâmetros utilizados nos experimentos foram baseados no alto nível de deformação que o material apresenta, sendo a velocidade de ensaio reduzida, de forma a não interferir nos resultados das propriedades elásticas do material em estudo. Para esse tipo de estudo o método mais adequado para a obtenção da força de ruptura é o de aplicação de carga estática através de ensaio mecânico de tração.

A avaliação tensiométrica revela-se bastante pertinente já que tem sido empregada em diversos estudos sobre cicatrização da parede abdominal. Compensa a subjetividade existente nos estudos sobre cicatrização em que apenas a avaliação histológica é empregada ${ }^{4,13,14}$.

$\mathrm{Na}$ avaliação microscópica utilizou-se apenas a coloração de hematoxilina-eosina. No estudo das peças o subgrupo Jatropha teve inflamação aguda mais intensa na avaliação do $3^{\circ}$ dia. A neoformação capilar mostrou-se estatisticamente significativa no subgrupo Jatropha do $3^{\circ}$ dia. Sendo o grau de inflamação aguda um fator determinante para uma cicatrização satisfatória, a sua exacerbação pode diminuir a irrigação sangüínea tecidual e comprometer a proliferação fibroblástica ${ }^{15,16,17}$.

Até o momento existem inúmeros estudos sobre Jatropha como: a) efeito neuromuscular e cardiovascular do tetramethilprazine, isolado da Jatropha podagrica; ${ }^{13}$ b) ação da Jatropha gossypiifolia L. sobre a contração da musculatura jejunal em ratos; ${ }^{14} \mathrm{c}$ ) experiência in vivo e in vitro do efeito farmacológico do extrato hidroalcoólico da Jatropha elíptica; ${ }^{20} \mathrm{~d}$ ) efeito antiadiarrêico significativo da Jatropha curcus, identificação e descrição de estruturas químicas de alcalóides, como imidazólicos e piperidínico ${ }^{18,19}$.

A continuação de pesquisas em fitoterapia utilizando-se a Jatropha, com estudos toxicológicos, utilizando-se extratos fracionados, é desafio a ser enfrentado. As plantas medicinais e os fitoterápicos adquirem importância como agentes terapêuticos e, por isso, devem ser prioritariamente analisados segundo os métodos mais modernos disponíveis ${ }^{20}$.

\section{Conclusão}

O uso do extrato bruto de Jatropha gossypiifolia L. intraperitoneal não demonstrou melhora significativa no processo de cicatrização da sutura da parede abdominal ventral de ratos com a dose e concentração utilizadas. 


\section{Referências}

1. Adolf W, Opferkuch HJ, Hecker. E. Irritant phorbol derivatives from Four Jatropha species. Phytochemistry $1984 ; 23: 129-32$.

2. Ahmad UM, Islam MR, Mirza PH. Alkaloides of Jatropha gossypiifolia Linn Indian J Chem. 1992; 31B: 67-9.

3. Corrêa MP. Dicionário das Plantas Úteis no Brasil e das Exóticas Cultivadas. Rio de Janeiro: Instituto Brasileiro de Desenvolvimento Florestal, 1984.

4. Fatureto, MC. Aspectos morfológicos do processo inflamatório provocado por fio de catgut simples no subcutâneo de ratos tratados com dicoflenaco sódico. [Dissertação - Mestrado] . São Paulo: Escola Paulista de Medicina. São Paulo; 1988.

5. Fernandez PM, Martins JL, Novo NF, Gomes PO, Goldenberg $\mathrm{S}$. Estudo comparativo da resistência à tração das cicatrizes nas laparotomias longitudinais e transversais em ratos. Acta Cir Bras. 1996; 11: 133-7.

6. Hodgson NC, Manthaner RA, Ostbye T. The search for an ideal method of abdominal fascial closure: a metaanalysis. Ann Surg 2000; 231: 436-42 .

7. Marchini FB, Martins DMFS, Teves DC, Simões MJ. Efeito do óleo de rosa mosqueta na cicatrização de feridas abertas. Rev Paul Med. 1998; 106: 356.

8. Teves DC, Cabral ACV, Simões MJ, Kulay Jr. L. Biologia da reparações teciduais. J Bras Med. 1986; 50:39-44

9. Mestriner F, Gomes RAS, Fatureto MC. Estudo comparativo entre as suturas contínuas e interrompidas da camada músculo fascial de ratos. Med Ribeirão Preto 1991; 24: 159-64.

10. Miller JM. Evaluation of a new cirurgical suture (prolene). Am Surg. 1973; 39: 31-9.

11. Minossi JG, Leite CVS, Naresse LE, Rodrigues MAM, Angeleli AYO, Kobayasi S. Efeito do diclofenaco de sódio na cicatrização da parede abdominal de ratos. Estudo histopatológico, da força de ruptura e do colágeno tecidual. Acta Cir Bras. 2001;16.

12. Rucinski J, Margolis M, Panagopoulos G, Wise L. Closure of the abdominal midline fascia: meta-analysis delineates the optimal technique. Am Surg. 2001; 67: 421-6.

13. Nair SK, Blat JK, Aurora A L. Role of proteolytic enzyme in the prevention of postoperative intraperitoneal adhesions. Arch Surg. 1974; 108: 849-53.

14. Seid MH, Mcdaniel-Owens LM,Poole Jr.GV. Meeks GR. A randomized trial of abdominal incision suture technique and wound strenght in rats. Arch Surg. 1995; 130: 394-7.

15. Ojewole JAO, Odebiyi O. Neuromuscular and Cardiovascular Actions of Tetramethylpyrazine from the Stem of Jatropha podagrica. J Med Plant Res. 1980; 38: 3328.

16. Simões MJ, Uzunian A, Mora AO, Sasso WS. Aspectos ultra-estruturais do processo de reparação da pele de ratos albinos. Rev Paul Med 1985; 103: 123-6.

17. Mantovani M, Leonard LS, Alcântara FG. Evolução da cicatrização em anastomoses do intestino grosso em condições de normalidade e sob ação de drogas imunossupressoras: estudo comparativo em cães. Rev Paul Med São Paulo 1997; 94; 118-26.

18. Paes AMA. Ação da Jatropha gossypiifolia L. (pião roxo) sobre a contração da musculatura lisa em ratos. [Monografia]. São Luis: Setor de Ciências Fisiológicas, Universidade Federal do Maranhão; 1997.

19. Tognini JRF, Fagundes DJ, Novo NF, Juliano Y. Estudo biomecênico e morfológico da cicatrização da parede abdominal sob ação de meloxicam. Acta Cir Bras. 2000; 15: $230-42$.

20. Trebien HA. Evaluation of Pharmacological activity of a crude hydroalcoholic extract from Jatropha elliptica. Phytoter Res. 1998; 2; 115-8.

\section{Correspondência}

José Ulcijara Aquino

Hospital Universitário Presidente Dutra

Rua Barão de Itapary, 227 - Centro - São Luis-MA

CEP: 65020-070

Tel: (98) 2109-1000
Conflito de interesse: nenhum Fonte de financiamento: Capes

Recebimento: 21/02/2005 Revisão: 03/06/2005 Aprovação: 27/06/2006

\section{Como citar este artigo}

Aquino JU, Czeczko NG, Malafaia O, Dietz UA, Ribas-Filho JM, Nassif PAN, Araújo U, Boroncello J, Santos MFS, Santos EAA. Avaliação fitoterápica da Jatropha gossypiifolia L. na cicatrização de suturas na parede abdominal ventral de ratos. Acta Cir Bras. [periódico na Internet] 2006; Suppl 2:61-66. Disponível em URL: http://www.scielo.br/acb

Figuras coloridas disponíveis em $\underline{\text { http://www.scielo.br/acb }}$ 